\title{
Structure-function relationships of phosphatidylinositol transfer proteins: involvement of phosphorylation sites
}

\author{
Gerry T. Snoek*, Claudia M. Van Tiel, Maarten R. Egmond \\ Department Biochemistry of Lipids, CBLE, University of Utrecht, Padualaan 8, De Uithof, Utrecht, The Netherlands
}

Received 26 August 2004; accepted 27 September 2004

Available online 19 October 2004

\begin{abstract}
The mammalian low molecular weight phosphatidylinositol transfer proteins: PI-TP $\alpha$ and PI-TP $\beta$ are extremely well conserved and highly homologous. Surprisingly, the two proteins clearly show different cellular localizations and display contrary physiological functions. Phosphorylation of the proteins might be the regulatory factor to ensure the selective cellular functions. A major difference between PI-TP $\alpha$ and PI-TP $\beta$ is the capacity of PI-TP $\beta$ to bind and transfer sphingomyelin (SM) in vivo. This activity is correlated with phosphorylation of Ser262, which is only present in PI-TP $\beta$. Structural aspects of phosphorylation sites of PI-TPs are analyzed in order to find an explanation for the functional data. We propose that phosphorylation of one serine residue (Ser165/166) in both PI-TP $\alpha$ and PI-TP $\beta$ is involved in the regulation of membrane binding of all PI-TPs and that phosphorylation of the unique Ser262 in PI-TP $\beta$-like proteins ensures the right cellular localization of PI-TP $\beta$ s that is necessary for the specific activity at the Golgi membrane.
\end{abstract}

(c) 2004 Elsevier SAS. All rights reserved.

Keywords: Phospholipid transfer proteins; Phosphatidylinositol transfer proteins; Protein kinase C; Crystal structure; Lipid transfer activity; Cellular localization

\section{Introduction}

Phosphatidylinositol transfer proteins (PI-TPs) belong to a family of highly conserved proteins that in vitro can catalyze the transfer of phosphatidylinositol (PI), phosphatidylcholine (PC) and sphingomyelin (SM) between membranes [1]. In mammalian tissues, at least two small molecular weight isoforms are identified: PI-TP $\alpha$ and PI-TP $\beta$. The isoforms demonstrate a high homology (77\%) and identity (91\%) [2]. Information about possible cellular functions of these proteins has been obtained from experiments with reconstituted Golgi membrane systems, permeabilized cells,

Abbreviations: $\mathrm{IP}_{3}$, inositol-3,4,5-trisphosphate; Nir, Protein Tyrosine kinase N-terminal domain interacting receptors; PC, phosphatidylcholine; PI, phosphatidylinositol; PIP, phosphatidylinositol-4-phosphate; $\mathrm{PIP}_{2}$, phosphatidylinositol-3,4-bisphosphate; PI-TP, phosphatidylinositol transfer proteins; PLA 2 , phospholipase $\mathrm{A}_{2}$; PLC, phospholipase C; PMA, phorbol, 12-myristate, 13-acetate; PS, phosphatidylserine; SM, sphingomyelin; $\mathrm{TNF} \alpha$, tumor necrosis factor $\alpha$.

* Corresponding author. Tel.: +31-30-253-2546; fax: +31-30-253-3151.

E-mail address: g.t.snoek@chem.uu.nl (G.T. Snoek). and from cells, tissues or animals in which the expression of the proteins has been manipulated.

Both proteins have been shown to stimulate the formation of secretory vesicles from isolated Golgi membranes as well as in permeabilized cells [3-5]. In permeabilized, cytosoldepleted HL60 cells both isoforms of PI-TP restored phospholipase C (PLC) mediated inositol lipid signaling [6,7]. However, other approaches emphasized that the PI-TP isoforms have separate cellular functions. Microinjection of fluorescently labeled PI-TP $\alpha$ and PI-TP $\beta$ into living cells as well as indirect immunofluorescence studies have shown that PI-TP $\alpha$ is localized in the nucleus and cytosol whereas PI$\mathrm{TP} \beta$ is associated with the Golgi system [8]. In intact cells, decreased expression of PI-TP $\alpha$ was shown to decrease the rate of proliferation and a lower rate of synthesis was observed for PC, lysoPC, SM, Choline and Glycerophosphocholine [9]. However, knock out of PI-TP $\alpha$ in mouse embryonic stem cells fails to compromise growth and has no significant consequence for bulk phospholipid metabolism. Moreover, the data showed that PI-TP $\alpha$ does not play an obvious role in any of the cellular activities where it has been reconstituted as an essential stimulatory factor. On the other 
hand, knock out of PI-TP $\beta$ in mouse embryonic stem cells was shown to be a fatal deletion [10]. Increased expression of PI-TP $\alpha$ in mouse fibroblast cells lead to an increased rate of proliferation and an increased cell survival upon induction of apoptosis by UV-light or tumor necrosis factor $\alpha$ (TNF $\alpha)$. The cellular effects were attributed to a PI-TP $\alpha$-dependent activation of a PI-specific phospholipase $\mathrm{A}_{2}\left(\mathrm{PLA}_{2}\right)$ since increased levels of lysoPI, Glycerophosphinositol, I(1)phosphate and I(2)phosphate were detected inside the cell [11]. In addition, highly mitogenic and anti-apoptotic arachidonic metabolites are secreted by the cells with increased expression of PI-TP $\alpha$. These, as yet unidentified secreted factors demonstrate autocrine as well as paracrine activity and most probably belong to the family of endocannabinoids [12-14]. Increased expression of PI-TP $\beta$ in mouse fibroblast cells increased the duration of the cell cycle and made these cells highly susceptible towards induction of apoptosis by UV-light or TNF $\alpha$ [15]. Finally, studies with intact animals produced interesting data. Mice carrying the vibrator mutation showed early onset, progressive tremor and degeneration of neurons in the brain stem and spinal cord followed by juvenile death. An $80 \%$ decreased expression of $\mathrm{PI}-\mathrm{TP} \alpha$ is responsible for the aberrant pathological condition; the level of PI-TP $\beta$ is unchanged [16]. PI-TP $\alpha$ knock out mice show a normal prenatal development but soon after birth start to show many neurodegenerative symptoms. It was found to be impossible to establish PI-TP $\beta$ knock out mice [17].

Little is known about the molecular mechanisms and regulatory processes that are involved in the described physiological events. It is not clear whether actual lipid transfer is involved in the physiological function of PI-TP $\alpha$ or PI-TP $\beta$ or how the binding/exchange of PI/PC/SM is established or regulated. Single or multiple phosphorylation of the two
PI-TP isoforms has been described and it has been shown that this phosphorylation is a regulatory factor for to the specific cellular localization, the lipid ligands bound and several of the cellular functions of the PI-TPs investigated $[15,18,19]$.

The crystal structure of PI-TP $\alpha$ without a phospholipid (the apo-form) as well as bound to a PC or a PI molecule have been described [20-22]. The structure of the apo-form was different from the PI- or PC-bound forms although the secondary structural elements are preserved. However, the overall fold of the PI- and PC-bound forms is highly similar, in contrast to previous suggestions [22]. In all current structures the main structural feature is a concave $\beta$-sheet consisting of eight strands (1-8) and seven $\alpha$-helices $(A-G)$. The part of the structure forming the lipid-binding site consists of the $\beta$-sheet en two $\alpha$-helices, $\mathrm{A}$ and $\mathrm{F}$ facing the interior of the $\beta$-sheet. In the apo-structure, the lipid-binding core is open; when a lipid is bound, helix $\mathrm{G}$ and loop $\mathrm{B}$ are moved over the lipid-binding core. The apo- and PI/PC-bound forms are shown in Fig. 1.

One of the putative membrane surface interaction sites of the molecule consists of a loop comprising two tryptophan residues, Trp203 and Trp204. The PI-TP $\alpha$ double mutant W203A/W204A is able to bind PI or PC but lacks PI/PC transfer activity and is unable to reconstitute PLC activity. The lipid head group cavity reveals a set of amino acids that can form H-bonds with inositol. The Choline head group is less well $\mathrm{H}$-bonded explaining the poor affinity for PC relative to PI [20].

The crystal structure of PI-TP $\beta$ has not yet been elucidated but because of the very high homology between PITP $\alpha$ and PI-TP $\beta$, a good model structure for PI-TP $\beta$ can be derived from that of the $\alpha$-species. Closer inspection of structural details reveals that only two amino acids are different in the phospholipid binding region: Ile84 and Phe225 in

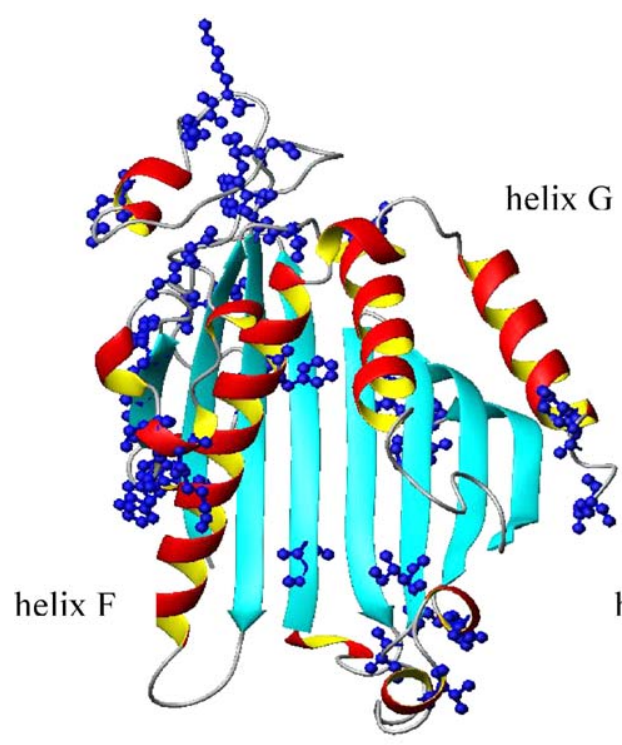

helix F

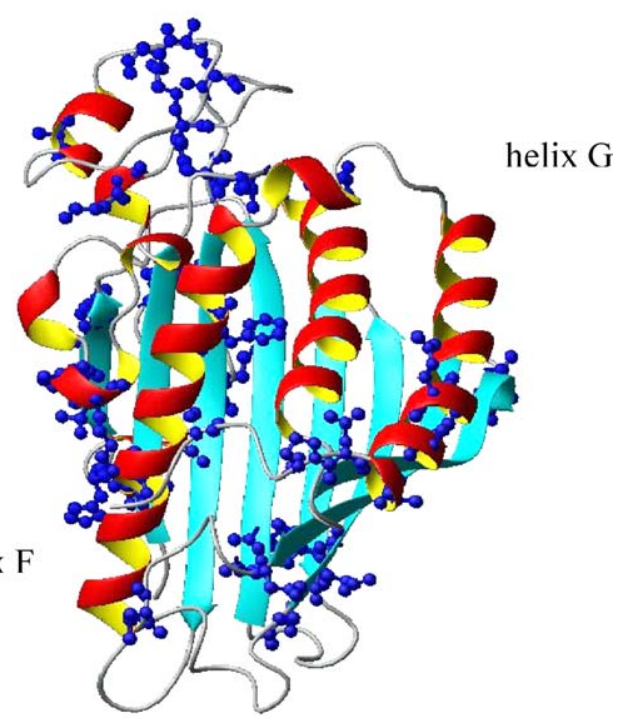

Fig. 1. The structures of apo- (left) and PI- or PC-bound PITP $\alpha$ (right) are shown indicating structural changes induced by phospholipid binding. Amino acid side chains are shown only for those 24 residues that are functionally different for the PI-TP $\alpha$ and PI-TP $\beta$ species. Most of these residues are not close to the phospholipid-binding site. 
PITP $\alpha$ are replaced by a Phe and Leu in the $\beta$-species, respectively. Only minor differences in substrate preference are to be expected from such subtle differences between the two forms-based on the properties of the substrate-binding region only.

In this paper we will review the data on phosphorylation of PI-TP $\alpha$ and PI-TP $\beta$ and the in vitro and in vivo consequences of selective phosphorylation. Based on these data we will propose a model for the relation between phosphorylation, protein structure and function as well as the regulation of activation and cellular localization by phosphorylation.

\section{Phosphorylation of PI-TP $\alpha$ and PI-TP $\beta$ in vitro and in vivo}

The specific capacity of PI-TPs to bind and transfer PI and PC between membranes formed the basis of the hypothesis that the proteins are involved in net transfer of PI (and exchange with PC) from the site of phospholipid synthesis, the Golgi network, to the plasma membrane. In the plasma membrane, PI is phosphorylated to phosphatidylinositol-4phosphate (PIP) by a PI-kinase and then to phosphatidylinositol-3,4-bisphosphate $\left(\mathrm{PIP}_{2}\right)$ by a PIP-kinase. Subsequently, upon stimulation of the cells $\mathrm{PIP}_{2}$ is degraded by PLC into inositol-3,4,5-trisphosphate $\left(\mathrm{IP}_{3}\right)$ and diacylglycerol (DAG). Two aspects of this metabolic pathway are of interest in the context of PI-TPs: the depletion of the PI pool in the plasma membrane by the stimulation of $\mathrm{PIP}_{2}$ degradation and the generation of DAG that activates protein kinase C [23]. Upon stimulation of PI-metabolism in Swiss Mouse 3T3 cells, the cellular localization of PI-TP, studied by indirect immunofluorescence, was changed. In contrast to what was expected, PI-TP did not move to the plasma membrane but it relocalized from the cytosol to the Golgi membranes. The Golgi localization was confirmed by double labeling with specific Golgi markers [24,25]. A comparable redistribution of PI-TP was observed when the cells were incubated with a direct activator of protein kinase $C$ (replacing DAG): phorbol, 12-myristate, 13-acetate (PMA). These results suggest the involvement of phosphorylation of PI-TP in the cellular relocalization. Metabolic labeling of mouse fibroblast cells with ${ }^{32} \mathrm{P}$-anorganic phosphate enabled the detection of phospho-PI-TP $\alpha$ but failed to show phospho-PITP $\beta$ because of the very low cellular concentration of PI-TP $\beta$ $[15,18,25]$. Using cells with increased expression of PI-TP $\beta$ and the use of very sensitive detection methods also allowed observation of the in vivo phosphorylation of PI-TP $\beta$ [19].

The amino acid sequences of mouse PI-TP $\alpha$ and PI-TP $\beta$ were analyzed for the presence of consensus sequences as putative phosphorylation sites that are specific for protein kinase $\mathrm{C}$. In PI-TP $\alpha$ one highly conserved serine residue can be phosphorylated by protein kinase C: Ser166. In PI-TP $\beta$ the same serine residue, $\mathrm{S} 165$, is a protein kinase $\mathrm{C}$-dependent phosphorylation site. A second serine residue in PI-TP $\beta$ that is phosphorylated by protein kinase C is Ser262.
An additional putative protein kinase $\mathrm{C}$-dependent phosphorylation site is Thr59 but PI-TP $\alpha$ or PI-TP $\beta$ are not phosphorylated on Thr59 when protein kinase $\mathrm{C}$ is stimulated by PMA or DAG nor upon in vitro phosphorylation by purified protein kinase C $[18,19,25]$.

Thr59 is very well conserved in the many members of the PI-TP family including the human Protein Tyrosine kinase $2 \mathrm{~N}$-terminal domain-interacting receptors (Nir2 and Nir3). The Nirs are membrane-bound proteins containing an N-terminal PI-TP-like domain [26]. Nirs are the human homologues of the Drosophila retinal degeneration B ( $\mathrm{RdgB})$ protein that is suggested to be involved in the fly photo transduction cascade in which the PI-TP domain is critical for its function [27-29]. A specific mutation of Thr59 in this domain induces a dominant retinal degeneration phenotype [27]. Nir2 is not an integral, membrane-spanning protein but is associated to Golgi/endoplasmatic reticulum membranes. A T59E mutation in Nir2 changed the cellular localization to spherical cytosolic lipid droplets [30]. It is suggested that phosphorylation of Thr59 and relocalization of Nir2 is stimulated by incubation with oleic acid. Since stimulation of protein kinase $\mathrm{C}$ by PMA had no apparent effect on Nir2 localization it was suggested that a phorbol ester-insensitive isoform of protein kinase $\mathrm{C}$ or some other Ser/Thr kinase induces threonine phosphorylation of Nir2 in response to oleic acid treatment [30].

Ser165/Ser166 and Thr59 are highly conserved through the large family of PI-TP-like proteins but Ser262 is only present in a few low molecular weight PI-TP $\beta$-like proteins (Table 1).

\subsection{In vitro phosphorylation of PI-TP $\alpha$ : kinetic analysis}

The first PI-TP that was purified to homogeneity from bovine brain had a molecular weight of $35 \mathrm{kDa}$ [31,32]. This protein was later denoted PI-TP $\alpha$. When this protein was first isolated from bovine brain cytosol two forms of PI-TP $\alpha$ were detected: PI-TP $\alpha$ I and PI-TP $\alpha$ II. These two forms could be separated because of a difference in isoelectric point, which was caused by the charge difference of the phospholipid species bound to the PI-TP $\alpha$. PI-TP $\alpha$ I has an isoelectric point of 5.5 (as determined under non-denaturing conditions) and was shown to have the negatively charged PI molecule in the lipid binding site of the protein, while PI-TP $\alpha$ II has an isoelectric point of 5.7 and was found to contain the zwitte-

Table 1

Members of the PI-TP $\beta$-family containing the Serine262 residue

\begin{tabular}{|c|c|}
\hline PI-TP $\beta$ rat & MRKKG S VRGTS \\
\hline PI-TP $\beta$ mouse & MRKKGG S VRGTS \\
\hline PI-TP $\beta$ human & MRKR $\bar{R} G \mathrm{~S}$ VRGTS \\
\hline PI-TPn-prov protein Xenopus laevis & MRQ $\bar{R} G$ T VRGMS \\
\hline zgc:55645 Danio rerio Table 3) & MRSQTG S VRGMK \\
\hline m-RdgB2 & KM̄AQF S EEGPS \\
\hline
\end{tabular}

$R$ : canonical consensus sequence for all known 10 isoforms of protein kinase C. K, R: canonical consensus sequence for conventional protein kinases $\mathrm{C}$ and for novel and a-typical protein kinases $\mathrm{C}$. 
rionic PC molecule [33]. The cellular concentration of PI$\mathrm{TP} \alpha \mathrm{I}$ is about eightfold higher as compared to the concentration of PI-TP $\alpha$ II. Since the affinity of PI-TP $\alpha$ for PC is about 16-fold lower than the affinity for PI, the relative amounts of the two charge isomers reflect the accessible pools of PI and $\mathrm{PC}$ in the cell $[33,34]$.

Recombinant murine PI-TP $\alpha$ and PI-TP $\alpha$ isolated from bovine brain were substrates for rat brain protein kinase $\mathrm{C}$ and were phosphorylated in a $\mathrm{Ca}^{2+}$ and a phosphatidylserine (PS) dependent manner in vitro [25,35]. Under optimal conditions, the calculated stoichiometry of the phosphorylation was $0.2 \mathrm{~mol}$ of phosphate/mol of PI-TP $\alpha$.

The charge isomers isolated from bovine brain were phosphorylated by protein kinase $\mathrm{C}$ at different rates. The estimated $V_{\max }$ values for PI-TP $\alpha$ I and PI-TP $\alpha$ II were 2.0 and $6.0 \mathrm{nmol} / \mathrm{min}$, respectively. The $K_{\mathrm{m}}$ values for both isomers were about equal, i.e. $0.7 \mu \mathrm{M}$ (Table 2, [18]). Phosphorylation of charge isomers of recombinant mouse PI-TP $\alpha$ confirmed that the PC-containing isomer was the better substrate. Phospho-amino acid analysis of in vitro and in vivo ${ }^{32} \mathrm{P}$-labeled PI-TPas showed that serine was the major site of phosphorylation. In addition, phosphorylated PI-TPaII contained a trace of phospho-threonine [18]. Replacement of Ser166 with an alanine residue confirmed that this serine residue is the site of phosphorylation. This mutation completely abolished PC and PI-transfer activity. This was also observed when Ser166 was replaced with an aspartic acid residue implying that the mere presence of a negatively charged amino acid residue is not sufficient for regulating the function of PI-TP $\alpha$ (Table 3).

Another putative protein kinase C-dependent phosphorylation site, Thr59, has been shown to play a crucial role in the activity/function of PI-TPs. Phosphorylation of threonine residues has not been observed either in in vivo or in vitro phosphorylated PI-TP $\alpha$. However, the trace of phospho-

Table 2

$K_{\mathrm{m}}$ and $V_{\max }$ values of the protein kinase C-dependent phosphorylation of $\mathrm{S} 166 / 165$ and S262 in PI-TP $\alpha$ and PI-TP $\beta$

\begin{tabular}{llc}
\hline & $K_{\mathrm{m}}(\mu \mathrm{M})$ & $V_{\max }(\mathrm{nmol} / \mathrm{min})$ \\
\hline Bovine brain PI-TP $\alpha \mathrm{I}$ & 0.65 & 2.0 \\
Bovine brain PI-TP $\alpha \mathrm{II}$ & 0.72 & 6.0 \\
Rec. PI-TP $\beta$ & 0.06 & 21.7 \\
Rec. PI-TP $\beta$ S165A & 0.06 & 24.6 \\
Rec. PI-TP $\beta$ S262A & 0.30 & 5.2 \\
\hline
\end{tabular}

Table 3

Lipid transfer activity of mutant PI-TP $\alpha$ and PI-TP $\beta$ proteins in vitro

\begin{tabular}{ll}
\hline Mutation & Lipid transfer activity \\
\hline$P I-T P \alpha$ & + \\
S152A & - \\
S166A & - \\
S166D & \\
$P I-T P \beta$ & - \\
S165A & + \\
S262A & - \\
S165A, S262A & - \\
\hline
\end{tabular}

threonine that was detected in phosphorylated PI-TP $\alpha$ II could represent Thr59 [18]. Phosphorylation of Thr59 by another threonine/serine kinase cannot be excluded [30].

\subsection{In vivo phosphorylation of PI-TP $\alpha$}

When PI-TP was immune-precipitated from cells that were labeled with ${ }^{32} \mathrm{P}$-inorganic phosphate, an increase in radioactivity in PI-TP from PMA-stimulated cells was observed leading to the conclusion that in stimulated cells, PI-TP is phosphorylated and redistributed in the cell [25]. Phospho-amino acid analysis of the in vivo phosphorylated PI-TP indicated exclusive phosphorylation on serine residues [18]. A complication in this conclusion is that at the time the existence of a highly homologous isoform of PI-TP was not yet known. Therefore, it is unclear whether the immuneprecipitated, phosphorylated protein represents phospho-PI$\mathrm{TP} \alpha$, phospho-PI-TP $\beta$ or a mixture of both. However, the 2-D-phosphopeptide map of in vivo phosphorylated PI-TP closely resembles the 2-D-phosphopeptide map of in vitro phosphorylated PI-TP $\alpha$ [19].

Upon PMA-stimulation of NIH3T3 mouse fibroblast cells transfected with cDNA encoding myc-tagged PI-TP $\alpha$, PI$\mathrm{TP} \alpha$ relocalization from the cytosol and nucleus to the Golgi membranes occurs [18]. In cells transfected with mutated PI-TP $\alpha$ : myc-PI-TP $\alpha(\mathrm{S} 166 \mathrm{~A})$ or myc-PI-TP $\alpha(\mathrm{S} 166 \mathrm{D})$, the myc-tagged mutant proteins were localized in the nucleus and the cytosol comparable to the myc-tagged wild type PI-TP $\alpha$. However, the mutant proteins did not relocalize upon stimulation by PMA suggesting that phosphorylation of S166 is required for relocalization to the Golgi membranes. Mouse fibroblast cells transfected with the mutant PITPaS166A demonstrated characteristics comparable to those of the wild type fibroblast cells and different from those of the fibroblast cells with increased expression of PI-TP $\alpha$ (unpublished results, $[11,18]$ ) indicating that the mutant PITP $\alpha$ failed to function as PI-TP $\alpha$ in the cell. Whether this is due to lack of phospholipid binding/transfer activity or to the missing phosphorylation site is yet unknown.

\subsection{In vitro phosphorylation of PI-TP $\beta$}

Unexpectedly it was found that PI-TP $\beta$ purified to homogeneity from bovine brain could not be phosphorylated by protein kinase $\mathrm{C}$ in vitro despite the presence of the same putative phosphorylation sites present in PI-TP $\alpha$ and even a few more (Table 1, [19,25]). On the other hand, mouse recombinant PI-TP $\beta$ was shown to be an excellent substrate for protein kinase $\mathrm{C}$ in vitro. The $V_{\max }$ and $K_{\mathrm{m}}$ values as shown in Table 2 confirmed this. The explanation was found when it was shown that in vivo PI-TP $\beta$ is already phosphorylated to a high level preventing additional phosphorylation of the native protein in vitro. PI-TP $\beta$ was exclusively phosphorylated on serine residues and mutation studies showed that Ser165 (comparable to Ser166 in PI-TP $\alpha$ ) is a minor phosphorylation site while Ser262 is a major phosphoryla- 
tion site [19]. Mutant PI-TP $\beta$ with Ser165 replaced by an alanine residue lost the capacity to transfer PI and PC in vitro as was shown for PI-TP $\alpha$. However, mutation of Ser262 had no effect on lipid transfer activities (Table 3 ).

\subsection{In vivo phosphorylation of PI-TP $\beta$}

In wild type mouse fibroblast cells, the level of PI-TP $\beta$ is too low to enable dependable studies on phosphorylation or cellular localization [15]. Mouse fibroblast cells with a 1015 -fold increased expression of PI-TP $\beta$ (SPI $\beta$ cells) showed significantly different physiological properties when compared to the wild type mouse fibroblast cells $[15,36]$ :

- The cell cycle duration increased from 21 to 34-36 h.

- The SM content of the plasma membrane was replenished more efficiently and rapidly upon degradation by exogenous SMase.

- SPI $\beta$ cells were extremely sensitive to apoptosis induced by UV-light or TNF $\alpha$.

In vivo phosphorylation of PI-TP $\beta$ could be studied by 2-D-gel electrophoresis followed by western blotting. PITP $\beta$ is detected as a $\mathrm{pH} 6.5$ form, phospho-PI-TP $\beta$ as a $\mathrm{pH}$ 6.2 form. In SPI $\beta$ cells more than $85 \%$ of PI-TP $\beta$ is phosphorylated. In cells transfected with a mutant PI-TP $\beta$, S262A, (SPI $\beta$ S262A cells) no phospho-PI-TP $\beta$ could be detected. Incubation of SPI $\beta$ cells with a protein kinase $C$ inhibitor, GF109203X, resulted in a shift from the phospho-PI-TP $\beta \mathrm{pH}$ 6.2 form to the PI-TP $\beta$ pH 6.5 form [19]. In SPI $\beta$ S262A cells the mutant PI-TP $\beta$ does not colocalize with the Golgi membranes as native PI-TP $\beta$ does [8] indicating a direct relationship between phosphorylation and cellular localization of PI-TP $\beta$. This was confirmed by the finding that incubation of SPI $\beta$ cells with the protein kinase C inhibitor GF109203X prevented the association of PI-TP $\beta$ with the Golgi membranes. The link between phosphorylation, localization and cellular function was given by the finding that SPI $\beta$ S262A cells fail to show the characteristics of SPI $\beta$ cells. The cell cycle duration, the sensitivity to induction of apoptosis by UV-light or TNF $\alpha$ and the replenishment of the degraded SM-pool in the plasma membrane in SPI $\beta$ S262A cells are comparable to those in wild type cells $[15,19]$. Since mutant PI-TP $\beta($ S262A) demonstrates the same in vitro lipid transfer activity as PI-TP $\beta$, it was concluded that the phosphorylation-dependent association of PI-TP $\beta$ with the Golgi membranes is essential for its cellular function. This indicates that Ser262 in members of the PI-TP $\beta$ family is necessary to direct the protein towards the Golgi membranes upon phosphorylation and that this event is essential for the correct functioning of the protein in the cell.

A psi BLAST analysis of proteins showing homology with PI-TP $\alpha$ and PI-TP $\beta$ indicated that Ser262 is only present in a few members of the PI-TP family (Table 1): in PI-TP $\beta$ from rat, mouse and human $[2,10,37]$. In zebrafish, a hypothetical protein derived from DNA sequences showed a high homology with PI-TP $\beta$ including the Ser262 with a protein kinase $\mathrm{C}$ consensus sequence [38]. In Xenopus a protein, PI-TPn was identified demonstrating a homology with PI-TP $\beta$. In PI-TPn a threonine residue is located on the 262 position instead of a serine residue but the surrounding amino acids form a consensus site for protein kinase C [39], Murine mRdgB2 (a high molecular weight, transmembrane member of the PI-TP family) contains a Ser262 but lacks the consensus properties [40].

\section{Structural aspects of phosphorylation of PI-TPa and PI-TPß}

The crystal structure of PI-TP $\alpha$ presents us the location of the putative protein kinase C-dependent phosphorylation sites in PI-TP $\alpha$ and PI-TP $\beta$, Ser165/Ser166, Ser262 and Thr59 [20-22]. As is shown in Fig. 2 a striking observation is the invariant position of Ser166 upon phospholipid binding [20]. The $\gamma-\mathrm{OH}$ of Ser166 is an unlikely target for phosphorylation in this orientation because it is not solvent accessible. Ser165/166 is found in a small pocket between the 165 and 172 loop and the remainder of the protein.

Several highly conserved residues anchor this loop including Lys168, Arg171, Asp234 and D238.

Based on these structural aspects it was concluded that for Ser166 to be phosphorylated, major conformational changes must occur such as may happen when PI-TP $\alpha$ is perturbed by interaction with either a membrane or a protein [20,41].

Fig. 3 shows the positions of Ser166 and another candidate for phosphorylation, Thr59, relative to a bound PI molecule.

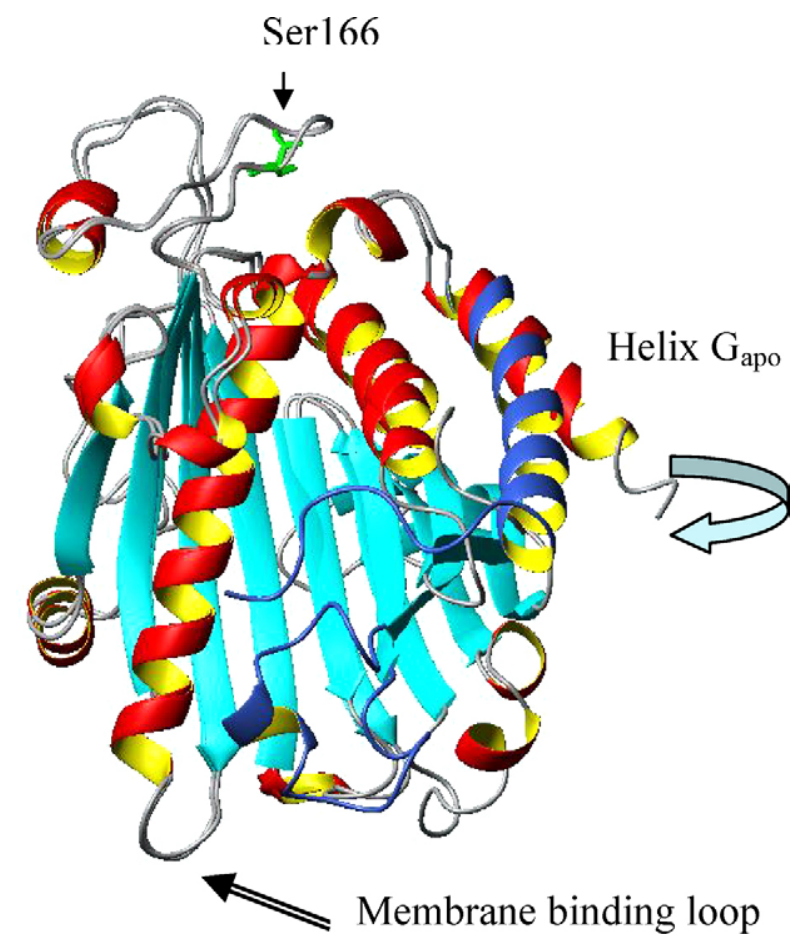

Fig. 2. Overlay of apo- and PI-bound PITP $\alpha$ species highlighting conformational changes induced by phospholipid binding, but also the invariant location of residue Ser166 and the membrane-binding loop containing Trp203 and Trp204. Changed conformations in loops and helices are colored blue in the PI-bound form only. 


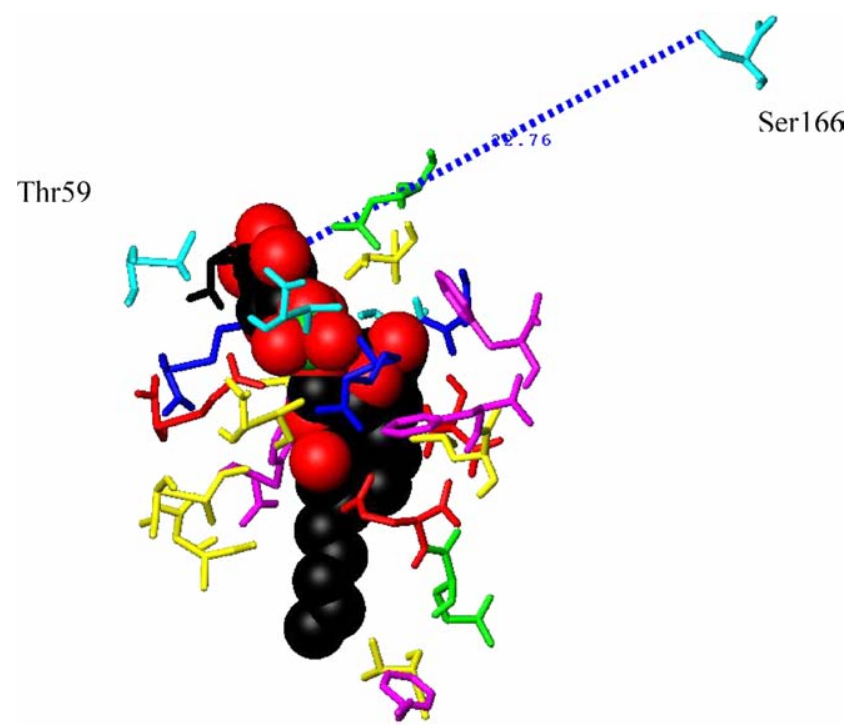

Fig. 3. Bound PI molecule surrounded by residues involved in substrate binding for PI-TP $\alpha$. In addition the location of Ser166 is shown relative to the substrate binding site.

The head group of PI is at a distance of about $23 \AA$ away from Ser166, which is in line with the lack of a reorientation of Ser166 upon PI binding. In contrast, Thr59 is directly involved in substrate recognition. Phosphorylation of Thr59 would certainly interfere with PI recognition (see more details below). The third residue mentioned, i.e. Ser262, is only present in PI-TP $\beta$. This residue is most likely accessible to solvent, and therefore, easily phosphorylated. However, the lack of a detailed model, particularly for PITP $\beta$ with bound substrate prevents more definite predictions to be made.

We propose the following model that will allow for a conformational change leading to phosphorylation of Ser166 after binding of PI-TP to a membrane: Helix F may slightly reorient when the loop at the $\mathrm{N}$-terminal side of this helix (involving Trp203 and Trp204) becomes embedded in the membrane. Ser166 is located at the C-terminal end of helix $\mathrm{F}$ above the helical axis at a distance of about $6 \AA$. This reorientation could trigger a slight conformational change leading to exposure of the $\gamma-\mathrm{OH}$ function of Ser166 allowing for this residue to become phosphorylated by protein kinase C.

This would predict that only membrane-bound PI-TP $\alpha$ is easily phosphorylated at Ser166. What about the presence or absence of bound substrate influencing these events? In vitro phosphorylation of PI-TP $\alpha$ showed that PI-TP $\alpha$ II was a better substrate for protein kinase $\mathrm{C}$ than PI-TP $\alpha$ I. The interactions of either lipid substrate, PI or PC, with the flanking helix F may modulate the forces that are needed to expose Ser166. The ligand in PI-TP $\alpha$ can either facilitate or inhibit this process explaining the different kinetics of phosphorylation of PI-TP $\alpha$ I and PI-TP $\alpha$ II [18]. In vitro phosphorylation of PI-TP $\alpha$ showed that the surface activity properties of the phosphorylated proteins change dramatically: the phosphorylated proteins bind quantitatively to the reaction vessels that are used for the phosphorylation reaction (plastic or glass) and could only be removed by detergents. The inability to recover native phosphorylated PI-TP $\alpha$ prevented the comparison of the lipid transfer activities of the phosphorylated and non-phosphorylated PI-TP $\alpha$ I and PI-TP $\alpha$ II (unpublished data G.T. Snoek).

Protein kinase C-dependent phosphorylation of Thr59 in PI-TP $\alpha$ from bovine brain or recombinant mouse PI-TP $\alpha$ has not been detected although a low level of phospho-threonine was found in phosphorylated PI-TP $\alpha$ II (the PC-carrying isomer) from bovine brain [18]. Replacement of Thr59 in rat PI-TP $\alpha$ by Ser, Gln, Val, Ile, Asn, Asp or Glu abolished PI-transfer activity but did not affect PC transfer activity [42]. This can be explained by the proposed models for PI-TP $\alpha$ where the inositol headgroup interacts directly with Thr59 whereas the choline headgroup does not [20,21]. Replacement of His60 by Q has no effect on either PI- or PC transfer activity. Thr59 is highly conserved among the family of PI-TPs, and therefore, may be of importance in the regulation of the protein activity. It is not yet known whether structural aspects or phosphorylation are involved in the regulatory role of Thr59 in the activity of PI-TP. In a high molecular weight member of the PI-TP family, Nir2, Thr59 can be phosphorylated suggesting the possibility that this residue can become solvent-accessible at some point during the lipid transfer cycle but that a different protein kinase is involved [30].

The lipid-binding site in the PI-TP $\beta$ model is virtually identical to the lipid-binding site in PI-TP $\alpha$. Mutation of Ser165 in PI-TP $\beta$ completely abolished phospholipid transfer activity, as was found also for PI-TP $\alpha[18,19]$. Since in PI-TP $\beta$, Ser165 is also phosphorylated by protein kinase C, we propose that regulation of the specificity and activity of PI-TP $\beta$ is also controlled by phosphorylation of Ser165 comparable to PI-TP $\alpha$.

A special feature of PI-TP $\beta$ is that it contains a second protein kinase C-dependent phosphorylation site: Ser262 that can be phosphorylated to a much higher level than S165 [19]. Mutation or phosphorylation of Ser262 has no effect on the lipid transfer activity in vitro. Phosphorylation of PI-TP $\beta$ on Ser262 was shown to be responsible for its cellular localization at the Golgi membranes. Microinjection of fluorescently labeled PI-TP $\beta$ isolated from bovine brain immediately localized to the Golgi membranes because, as was shown later, native PI-TP $\beta$ is already phosphorylated to a very high level $[8,19]$.

\section{Conclusions and discussion}

As in vivo phosphorylation of Ser166 is strongly correlated with the binding to a membrane surface, the phosphorylation and dephosphorylation cycle of Ser166 could regulate the specificity and the rate of lipid binding and/or transfer by PI-TP $\alpha$ and/or PI-TP $\beta$.

Stimulation of phosphorylation of Ser166 in PI-TP $\alpha$ mediates a translocation of PI-TP $\alpha$ to the Golgi membranes and 
vesicle-like structures. However, since the cellular functions of PI-TP $\alpha$ and PI-TP $\beta$ have been shown to be clearly distinct, it must be concluded that Golgi-association of PI-TP $\alpha$ serves a different purpose than Golgi association of PI-TP $\beta$. It is not known yet whether the Golgi-association of PI-TP $\alpha$ is necessary for its cellular function. Expression of PI-TPaS166A in mouse fibroblast cells has no effect on growth characteristics in contrast to an increased expression of PI-TP $\alpha$. Whether this lack of effect is caused by the absence of the phosphorylation site in PI-TP $\alpha$ S166A or by the biological inactivity of the expressed protein remains to be established.

Phosphorylation of Ser262 is restricted to PI-TP $\beta$-like proteins and its major function seems to be the regulation of the cellular localization at the Golgi membranes. This localization nevertheless is essential for the adequate cellular functioning of PI-TP $\beta$. Expression of PI-TP $\beta$ S262A in mouse fibroblast has no effect on growth characteristics of the cells in contrast to increased expression of PI-TP $\beta$. This difference can be contributed to the absence of the phosphorylation site in PI-TP $\beta$ S262A since the protein is biologically active (in vitro).

Major differences between PI-TP $\alpha$ and PI-TP $\beta$ are the capacity of PI-TP $\beta$ to bind and transfer SM and the presence of Ser262 in PI-TP $\beta$ regulating its essential Golgi localization. Thus, phosphorylation/dephosphorylation of one conserved serine residue (Ser165/166) in both PI-TP $\alpha$ and PITP $\beta$ seems to be involved in regulation of membrane binding of all PI-TPs and phosphorylation of the unique Ser262 in PI-TP $\beta$-like proteins seems to ensure the right cellular localization of these proteins that is necessary specific activity at the Golgi membrane.

\section{References}

[1] K.W. Wirtz, Phospholipid transfer proteins revisited, Biochem. J. 324 (Pt. 2) (1997) 353-360.

[2] S. Tanaka, K. Hosaka, Cloning of a cDNA encoding a second phosphatidylinositol transfer protein of rat brain by complementation of the yeast sec14 mutation, J. Biochem. (Tokyo) 115 (1994) 981-984.

[3] M. Ohashi, K. Jan de Vries, R. Frank, G. Snoek, V. Bankaitis, $\mathrm{K}$. Wirtz, et al., A role for phosphatidylinositol transfer protein in secretory vesicle formation, Nature 377 (1995) 544-547.

[4] S.M. Jones, J.G. Alb Jr., S.E. Phillips, V.A. Bankaitis, K.E. Howell, A phosphatidylinositol 3-kinase and phosphatidylinositol transfer protein act synergistically in formation of constitutive transport vesicles from the trans-Golgi network, J. Biol. Chem. 273 (1998) 1034910354.

[5] J.C. Hay, T.F. Martin, Phosphatidylinositol transfer protein required for ATP-dependent priming of $\mathrm{Ca}(2+)$-activated secretion, Nature 366 (1993) 572-575.

[6] G.M. Thomas, E. Cunningham, A. Fensome, A. Ball, N.F. Totty, O. Truong, et al., An essential role for phosphatidylinositol transfer protein in phospholipase C-mediated inositol lipid signaling, Cell 74 (1993) 919-928.

[7] K.J. de Vries, A.A. Heinrichs, E. Cunningham, F. Brunink, J. Westerman, P.J. Somerharju, et al., An isoform of the phosphatidylinositoltransfer protein transfers sphingomyelin and is associated with the Golgi system, Biochem. J. 310 (Pt. 2) (1995) 643-649.
[8] K.J. De Vries, J. Westerman, P.I. Bastiaens, T.M. Jovin, K.W. Wirtz, G.T. Snoek, Fluorescently labeled phosphatidylinositol transfer protein isoforms (alpha and beta), microinjected into fetal bovine heart endothelial cells, are targeted to distinct intracellular sites, Exp. Cell Res. 227 (1996) 33-39.

[9] M.E. Monaco, R.J. Alexander, G.T. Snoek, N.H. Moldover, K.W. Wirtz, P.D. Walden, Evidence that mammalian phosphatidylinositol transfer protein regulates phosphatidylcholine metabolism, Biochem. J. 335 (Pt. 1) (1998) 175-179.

[10] J.G. Alb Jr., S.E. Phillips, K. Rostand, X. Cui, J. Pinxteren, L. Cotlin, T. Manning, S. Guo, J.D. York, H. Sontheimer, J.F. Collawn, V.A. Bankaitis, Genetic ablation of phosphatidylinositol transfer protein function in murine embryonic stem cells, Mol. Biol. Cell 13 (2002) 739-754.

[11] G.T. Snoek, C.P. Berrie, T.B. Geijtenbeek, H.A. Van der Helm, J.A. Cadee, C. Iurisci, et al., Overexpression of phosphatidylinositol transfer protein alpha in NIH3T3 cells activates a phospholipase A, J. Biol. Chem. 274 (1999) 35393-35399.

[12] M. Schenning, C.M. Van Tiel, D. Van Manen, J. Stam, B.M. Gadella, K.W.A. Wirtz, G.T. Snoek, Phosphatidylinositol transfer protein \{alpha\} regulates growth and apoptosis of NIH3T3 cells: involvement of a cannabinoid 1-like receptor, J. Lipid Res. 45 (2004) 1555-1564.

[13] D. Piomelli, M. Beltramo, A. Giuffrida, N. Stella, Endogenous cannabinoid signaling, Neurobiol. Dis. 5 (1998) 462-473.

[14] D. Piomelli, The molecular logic of endocannabinoid signalling, Nat. Rev. Neurosci 4 (2003) 873-884.

[15] C.M. Van Tiel, M. Schenning, G.T. Snoek, K.W.A. Wirtz, Overexpression of phosphatidylinositol transfer protein beta in NIH3T3 cells has a stimulatory effect on sphingomyelin synthesis and apoptosis, Biochim. Biophys. Acta 1636 (2004) 151-158.

[16] B.A. Hamilton, D.J. Smith, K.L. Mueller, A.W. Kerrebrock, R.T. Bronson, V. Van Berkel, et al., The vibrator mutation causes neurodegeneration via reduced expression of PITP alpha: positional complementation cloning and extragenic suppression, Neuron 18 (1997) 711-722.

[17] J.G. Alb Jr., J.D. Cortese, S.E. Phillips, R.L. Albin, T.R. Nagy, B.A. Hamilton, V.A. Bankaitis, Mice lacking phosphatidylinositol transfer protein-alpha exhibit spinocerebellar degeneration, intestinal and hepatic steatosis, and hypoglycemia, J. Biol. Chem. 278 (2003) 33501-33518.

[18] C.M. Van Tiel, J. Westerman, M. Paasman, K.W. Wirtz, G.T. Snoek, The protein kinase C-dependent phosphorylation of serine 166 is controlled by the phospholipid species bound to the phosphatidylinositol transfer protein alpha, J. Biol. Chem. 275 (2000) 2153221538.

[19] C.M. Van Tiel, J. Westerman, M.A. Paasman, M.M. Hoebens, K.W. Wirtz, G.T. Snoek, The Golgi localization of phosphatidylinositol transfer protein beta requires the protein kinase C-dependent phosphorylation of serine 262 and is essential for maintaining plasma membrane sphingomyelin levels, J. Biol. Chem. 277 (2002) 2244722452.

[20] S.J. Tilley, A. Skippen, J. Murray-Rust, P.M. Swigart, A. Stewart, C.P. Morgan, S. Cockcroft, N.Q. McDonald, Structure-function analysis of phosphatidylinositol transfer protein alpha bound to human phosphatidylinositol, Structure (Cambridge) 12 (2004) 317326.

[21] M.D. Yoder, L.M. Thomas, J.M. Tremblay, R.L. Oliver, L.R. Yarbrough, G.M. Helmkamp Jr., Structure of a multifunctional protein. Mammalian phosphatidylinositol transfer protein complexed with phosphatidylcholine, J. Biol. Chem. 276 (2001) 9246-9252.

[22] A. Schouten, B. Agianian, J. Westerman, J. Kroon, K.W. Wirtz, P. Gros, Structure of apo-phosphatidylinositol transfer protein alpha provides insight into membrane association, EMBO J. 21 (2002) 2117-2121.

[23] A.C. Newton, Diacylglycerol's affair with protein kinase C turns 25 , Trends Pharmacol. Sci. 25 (2004) 175-177. 
[24] G.T. Snoek, I.S. de Wit, J.H. Van Mourik, K.W. Wirtz, The phosphatidylinositol transfer protein in 3T3 mouse fibroblast cells is associated with the Golgi system, J. Cell. Biochem. 49 (1992) 339-348.

[25] G.T. Snoek, J. Westerman, F.S. Wouters, K.W. Wirtz, Phosphorylation and redistribution of the phosphatidylinositol-transfer protein in phorbol 12-myristate 13-acetate- and bombesin-stimulated Swiss mouse 3 T3 fibroblasts, Biochem. J. 291 (Pt. 2) (1993) 649-656.

[26] S. Lev, J. Hernandez, R. Martinez, A. Chen, G. Plowman, J. Schlessinger, Identification of a novel family of targets of PYK2 related to Drosophila retinal degeneration B ( $\mathrm{RdgB})$ protein, Mol. Cell. Biol. 19 (1999) 2278-2288.

[27] S.C. Milligan, J.G. Alb Jr., R.B. Elagina, V.A. Bankaitis, D.R. Hyde, The phosphatidylinositol transfer protein domain of Drosophila retinal degeneration B protein is essential for photoreceptor cell survival and recovery from light stimulation, J. Cell Biol. 139 (1997) 351-363.

[28] C.T. Rubinstein, S. Bar-Nachum, Z. Selinger, B. Minke, Lightinduced retinal degeneration in retinal degeneration $\mathrm{B}(\mathrm{RdgB})$ mutant of Drosophila: electrophysiological and morphological manifestations of degeneration, Vis. Neurosci. 2 (1989) 529-539.

[29] W.A. Harris, W.S. Stark, Hereditary retinal degeneration in Drosophila melanogaster. A mutant defect associated with the phototransduction process, J. Gen. Physiol. 69 (1977) 261-291.

[30] V. Litvak, Y.D. Shaul, M. Shulewitz, R. Amarilio, S. Carmon, S. Lev, Targeting of Nir2 to lipid droplets is regulated by a specific threonine residue within its PI-transfer domain, Curr. Biol. 12 (2002) 15131518.

[31] P.A. Van Paridon, A.J. Visser, K.W. Wirtz, Binding of phospholipids to the phosphatidylinositol transfer protein from bovine brain as studied by steady-state and time-resolved fluorescence spectroscopy, Biochim. Biophys. Acta 898 (1987) 172-180.

[32] G.M. Helmkamp Jr., S.A. Nelemans, K.W.A. Wirtz, Immunological comparison of phosphatidylinositol and phosphatidylcholine exchange proteins in bovine brain, liver and heart, Biochim. Biophys. Acta 424 (1976) 168-182.

[33] P.A. Van Paridon, T.W. Gadella Jr., P.J. Somerharju, K.W. Wirtz, On the relationship between the dual specificity of the bovine brain phosphatidylinositol transfer protein and membrane phosphatidylinositol levels, Biochim. Biophys. Acta 903 (1987) 68-77.

[34] R.A. Demel, R. Kalsbeek, K.W. Wirtz, L.M. Van Deenen, The proteinmediated net transfer of phosphatidylinositol in model systems, Biochim. Biophys. Acta 466 (1977) 10-22.

[35] T.B. Geijtenbeek, E. de Groot, J. Van Baal, F. Brunink, J. Westerman, G.T. Snoek, et al., Characterization of mouse phosphatidylinositol transfer protein expressed in Escherichia coli, Biochim. Biophys. Acta 1213 (1994) 309-318.
[36] C.M. Van Tiel, C. Luberto, G.T. Snoek, Y.A. Hannun, K.W. Wirtz, Rapid replenishment of sphingomyelin in the plasma membrane upon degradation by sphingomyelinase in NIH3T3 cells overexpressing the phosphatidylinositol transfer protein beta, Biochem. J. 346 (Pt. 2) (2000) 537-543.

[37] S. Tanaka, S. Yamashita, K. Hosaka, Cloning and expression of human cDNA encoding phosphatidylinositol transfer protein beta, Biochim. Biophys. Acta 1259 (1995) 199-202.

[38] R.L. Strausberg, E.A. Feingold, L.H. Grouse, J.G. Derge, R.D. Klausner, F.S. Collins, L. Wagner, C.M. Shenmen, G.D. Schuler, S.F. Altschul, B. Zeeberg, K.H. Buetow, C.F. Schaefer, N.K. Bhat, R.F. Hopkins, H. Jordan, T. Moore, S.I. Max, J. Wang, F. Hsieh, L. Diatchenko, K. Marusina, A.A. Farmer, G.M. Rubin, L. Hong, M. Stapleton, M.B. Soares, M.F. Bonaldo, T.L. Casavant, T.E. Scheetz, M.J. Brownstein, T.B. Usdin, S. Toshiyuki, P. Carninci, C. Prange, S.S. Raha, N.A. Loquellano, G.J. Peters, R.D. Abramson, S.J. Mullahy, S.A. Bosak, P.J. McEwan, K.J. McKernan, J.A. Malek, P.H. Gunaratne, S. Richards, K.C. Worley, S. Hale, A.M. Garcia, L.J. Gay, S.W. Hulyk, D.K. Villalon, D.M. Muzny, E.J. Sodergren, X. Lu, R.A. Gibbs, J. Fahey, E. Helton, M. Ketteman, A. Madan, S. Rodrigues, A. Sanchez, M. Whiting, A.C. Young, Y. Shevchenko, G.G. Bouffard, R.W. Blakesley, J.W. Touchman, E.D. Green, M.C. Dickson, A.C. Rodriguez, J. Grimwood, J. Schmutz, R.M. Myers, Y.S. Butterfield, M.I. Krzywinski, U. Skalska, D.E. Smailus, A. Schnerch, J.E. Schein, S.J. Jones, M.A. Marra, Generation and initial analysis of more than 15,000 full-length human and mouse cDNA sequences, Proc. Natl. Acad. Sci. USA 99 (2002) 16899-16903.

[39] S.L. Klein, R.L. Strausberg, L. Wagner, J. Pontius, S.W. Clifton, P. Richardson, Genetic and genomic tools for Xenopus research: the NIH Xenopus initiative, Dev. Dyn. 225 (2002) 384-391.

[40] C. Lu, T.S. Vihtelic, D.R. Hyde, T. Li, A neuronal-specific mammalian homolog of the Drosophila retinal degeneration B gene with expression restricted to the retina and dentate gyrus, J. Neurosci. 19 (1999) 7317-7325.

[41] C.M. Van Tiel, A. Schouten, G.T. Snoek, P. Gros, K.W. Wirtz, The structure of phosphatidylinositol transfer protein alpha reveals sites for phospholipid binding and membrane association with major implications for its function, FEBS Lett. 531 (2002) 69-73.

[42] J.G. Alb Jr., A. Gedvilaite, R.T. Cartee, H.B. Skinner, V.A. Bankaitis, Mutant rat phosphatidylinositol/phosphatidylcholine transfer proteins specifically defective in phosphatidylinositol transfer: implications for the regulation of phospholipid transfer activity, Proc. Natl. Acad. Sci. USA 92 (1995) 8826-8830. 